Research Article

\title{
Assessment of Lifetime Attributable Risk for Public Health Sustainability from the Fukushima Accident
}

\author{
Faith Rukundo and Juyoul Kim \\ Department of NPP Engineering, KEPCO International Nuclear Graduate School, 658-91 Haemaji-ro, Seosaeng-myeon, \\ Ulju-gun, Ulsan 45014, Republic of Korea \\ Correspondence should be addressed to Juyoul Kim; jykim@kings.ac.kr
}

Received 13 July 2020; Accepted 23 October 2020; Published 4 November 2020

Academic Editor: Arkady Serikov

Copyright (C) 2020 Faith Rukundo and Juyoul Kim. This is an open access article distributed under the Creative Commons Attribution License, which permits unrestricted use, distribution, and reproduction in any medium, provided the original work is properly cited.

\begin{abstract}
The study aimed at reassessing the radiological risk from exposure to ionizing radiation from Fukushima Daiichi nuclear power plant accident. The estimated risks from the study were compared with World Health Organization (WHO) risk assessment estimates for validity and verification. A Radiation Risk Assessment Tool (RadRAT) was used to estimate Lifetime Attributable Risks (LAR) of cancer upon exposure. All solid cancers, leukemia, and thyroid cancer risks for ages of 1, 10, and 20 years (infants, children, and adults) in 100,000 persons at exposure were estimated. For solid cancers, LAR $\left(10^{-2}\right)$ was estimated in a range of $0.223 \sim 0.668$ and $0.345 \sim 1.24$ for males and females, respectively, whereas the LAR $\left(10^{-2}\right)$ for leukemia was estimated at $0.0155 \sim 0.055$ and $0.0118 \sim 0.0375$ for males and females, respectively. LAR $\left(10^{-2}\right)$ for thyroid cancer ranged from $0.0722 \sim 0.545$ and $0.0369 \sim 0.265$, respectively.
\end{abstract}

\section{Introduction}

A nuclear accident is described as an event that leads to a radiation release and resultant significant consequences for the general public, environment, and nuclear facility where it occurred [1]. Deterministic (short term) and stochastic (long term) effects are associated with exposure to dose by ionizing radiation. At lower doses, stochastic effects, particularly somatic effects (cancer), are expected to occur and are basically proportional to exposure doses, while at higher doses, deterministic effects occur, and there is a threshold of doses below which the effect from exposure does not occur [2]. Understanding the relationship between exposure to radiation dose and the health risk effect is very necessary for the proper emergency planning and response immediately after a nuclear power plant (NPP) accident. Generally, the informative epidemiological data on human exposure to radiation has been described from the life span study (LSS) of Japanese atomic bomb survivors who were exposed to radiation at Hiroshima and Nagasaki in 1945 [3]. Nuclear accidents lead to the release of many different radioactive materials that have different toxic effects depending on the type of radiation such as gamma ray, beta, and alpha particles. Researches on the previous accidents at NPPs have suggested that inert gases including xenon and krypton are likely to be released along with iodine, cesium, strontium, tellurium, and rubidium [4]. The Fukushima Daiichi NPP accident that occurred on March 11, 2011 released a large amount of radioactive materials into the environment. Volatile fission products including noble gases of xenon $\left({ }^{133} \mathrm{Xe}\right)$ and krypton $\left({ }^{85} \mathrm{Kr}\right)$, isotopes of cesium $\left({ }^{134} \mathrm{Cs},{ }^{136} \mathrm{Cs}\right.$, $\left.{ }^{137} \mathrm{Cs}\right)$ and iodine $\left({ }^{131} \mathrm{I},{ }^{132} \mathrm{I}\right)$, and tellurium $\left({ }^{132} \mathrm{Te}\right)$ were found to be dominantly released [5]. ${ }^{131}$ I was considered as one of the most important radionuclides during the first week after a nuclear accident due to its short half-life of 8 days. Doses to the thyroid gland require special attention because of their contributions to the total effective dose from short-lived (mainly, ${ }^{131} \mathrm{I}$ ) and long-lived (mainly, ${ }^{134} \mathrm{Cs}$ and ${ }^{137} \mathrm{Cs}$ ) radionuclides [6]. The current methodology and approach to the nuclear emergency planning and response adopted in most countries in the world are generally deterministic based on the dose estimation of off-site 
radiological consequences. But it has been getting more attention and importance to provide and implement the probabilistic risk-informed technical supports in decisionmaking processes related with NPP emergency planning and response measures since Fukushima nuclear accidents in 2011 [7-9]. This study investigated the reassessment of the radiological risks from exposure to the radioactive releases from the Fukushima Daiichi nuclear accidents during the first four days of the accident. Following the available radiological source terms, the radiological assessment system for consequence analysis (RASCAL) has been applied to evaluate the doses and assess the lifetime attributable risk (LAR) of homogeneous population groups affected by the accident [10-13]. Cancer risk assessment is still challenging in the radiation-related industries and hospital activities, and thus this research will be relevant to reemphasize the need to understand and predict the cancer risk that may occur after exposure to a large amount of radiation from a nuclear accident. With the help of different methods and assumptions, the cancer risk estimates in this study were compared with those of World Health Organization (WHO) study in 2013 to check their validity and correlation. The relationship between radiation exposure and lifetime risk of cancer was investigated using some factors including radiation dose, age at exposure, sex, and cancer site.

\section{Materials and Methods}

2.1. Radiological Assessment System for Consequence Analysis (RASCAL). RASCAL was developed for the Nuclear Regulatory Commission (NRC) as a tool for the rapid assessment of an incident or accident in order to help a decision-making process of implementing protective actions such as evacuation and sheltering in the first four days after a nuclear accident. Following the same accident sequence of Fukushima Daiichi nuclear accident, RASCAL was used to estimate the radiological source terms and radiation doses. The source term to dose module (STDose) in RASCAL was used to predict the total effective dose equivalent (TEDE) and thyroid dose within a radius of 1.61 to $32 \mathrm{~km}$ from the plume of the released radioactive materials to the downwind public where the wind is blowing towards for 96 hours [14]. The STDose module generated time-dependent source terms and then provided the input data to the atmospheric transport and dispersion model. STDose module required information such as event location, event type, release path, source term, and meteorological data in order to predict the exposure dose to the general public. Gaussian plume and Gaussian puff models are embedded within RASCAL for the atmospheric transport, dispersion, and deposition of radioactive materials from the release point. The conventional straight-line Gaussian equation used in RASCAL was as follows:

$$
\chi(x, y, x)=\int_{-\infty}^{\infty} \frac{Q^{\prime} F_{y} F_{z}}{(2 \pi)^{3 / 2} \sigma_{x} \sigma_{y} \sigma_{z}} \exp \left[\frac{1}{2}\left(\frac{x-u t}{\sigma_{x}}\right)^{2}\right] \mathrm{d} t
$$

A simplified version of the straight-line Gaussian model could be expressed by

$$
\frac{\chi(x, y, z)}{Q^{\prime}}=\frac{F_{y} F_{z}}{2 \pi u \sigma_{y} \sigma_{z}},
$$

where $\chi=$ mean concentration, $Q^{\prime}=$ release rate, $F_{y}, F_{z}=$ lateral and vertical exponential terms, $x=$ downwind distance at which $\chi, \sigma_{x}, \sigma_{y}$ and $\sigma_{z}$ are evaluated, $u=$ speed of the wind, $t=$ time.

Using the feature of multi-unit source term merge in RASCAL, the merging of atmospheric source terms from multiple units on a single NPP site provided a means to calculate doses from radioactive releases of Fukushima Daiichi reactors. The technique assumed that the releases occurred at the same location; however, the individual release points might be separated by as much as several hundred feet. The separation might cause the results near the release point to be in error, but it might not necessarily have a considerable effect on the results at off-site locations.

2.2. Radiation Risk Assessment Tool (RadRAT). RadRAT, an online tool that was developed by the national cancer institute of United States, was used in this study to estimate the lifetime attributable risks (LAR) of radiation-related cancers. RadRAT enables users to estimate the lifetime risk of cancer incidence for the general population of United States and some other selected countries from exposure to ionizing radiation in terms of radiation doses below $1 \mathrm{~Sv}$. RadRAT followed the biologic effects of ionizing radiation (BEIR) VII report assumptions by applying an uncertain dose and doserate effectiveness factor (DDREF) for all chronic exposures using a lognormal distribution with a geometric mean of 1.5 and no-threshold risk models [15]. Input information that was required by RadRAT running included sex, birth year, history of exposure, and run-specific parameters. RadRAT 4.1.1 was used in this study because it followed the methodology of WHO framework very closely and included the 2010 Japanese population data. The radiation risk calculator estimated LAR from the time when an individual was exposed until the end of expected lifetime. Future risks were given as risk attributable to radiation (i.e., the excess risk), the baseline risk (i.e., risk in the absence of exposure to radiation), and total risk (i.e., excess plus baseline). LAR calculation was performed as a function of attained age $(a)$ for the period of life after radiation exposure up to the end of the $90^{\text {th }}$ year of life. LAR from one yearly dose $(\operatorname{LAR}(D, e, s))$ specifies the sex $(s)$, age-at-exposure (e), and specific cumulative probability of a specific cancer attributable to radiation exposure over a period up to a maximum age $\left(a_{\max }\right)$ :

$$
\operatorname{LAR}(D, e, s)=\int_{e+L}^{a_{\max }} M(D, e, a, s) \frac{S_{a j}(a, g)}{S_{a j}(e, g)} \mathrm{d} a,
$$

where, $M(D, e, a, s)$ is the risk model, $S_{a j}(a, g)$ is the probability of surviving cancer-free to age $(a)$ for the unexposed population, $L$ is the minimum latency period, and the ratio $S_{a j}(a, g) / S_{a j}(e, g)$ is the conditional probability of a 
person alive and cancer-free at age-at exposure $(e)$ to reach at least an attained age $(a)$.

\section{Results and Discussion}

3.1. Source Term and Dose Estimation. Using RASCAL 4.3.3, this study used previously estimated $1.3 \times 10^{18} \mathrm{~Bq}$ by the authors as the total amount of source terms released from the Fukushima Daiichi NPPs to the atmospheric environment [14]. In the authors' previous study, estimated source terms were quantitatively compared and discussed with the existing literatures, which had been derived from the estimation method using accident progression and inverse modeling by US Nuclear Regulatory Commission, International Atomic Energy Agency, Nuclear and Industry Safety Agency in Japan, and other four individual researchers in the world. Although the model comparison showed a good fit within the same order of magnitude in terms of activities of ${ }^{131} \mathrm{I}$ and ${ }^{137} \mathrm{Cs}$, it was found that their differences were mainly attributed to the assumed values of leakage rates from the reactor containment to the environment, mathematical models embedded in different computer codes, and uncertainties of values measured at the time of the accident. The simulation was conducted for a period from $12^{\text {th }}$ to $15^{\text {th }}$ March 2011 because it was the period during which most radioactive materials were released to the environment. The determined dose values were applied to estimate the radiological health risks that might have been incurred by the Japanese people. The TEDE was calculated as the summation of inhalation committed effective dose equivalent (CEDE), cloud-shine, and 4-day ground-shine. The inhalation CEDE was calculated to be a 50 -year committed dose to an adult. Table 1 provides a summary of reference doses for individuals exposed from NPPs to up to a distance of $32.2 \mathrm{~km}$ during the first 4 days.

3.2. Cancer Risk Estimation. The LAR of developing cancer for the general population in Japan consisting of both males and females exposed at 1 year (infant), 10 years (children), and 20 years of age (adults) was estimated using RadRAT. The LAR in this study was defined as the probability of an early incidence of a cancer attributable to radiation exposure in a representative group of the population. A sample size of 100,000 persons at exposure was applied in this study. Due to their unique radio-sensitivity, solid cancers, thyroid cancer, and leukemia were estimated using the population status of Japan in 2010. Based on cancer incidence rates from a general population, the lifetime baseline risk (LBR) was estimated as the cumulated baseline probability of having a specific cancer over the lifetime (up to the age of 89 years). Tables 2 and 3 show the LAR of developing solid cancers, leukemia, and thyroid cancer for females and males of different ages in 100,000 persons exposed to a certain amount of radiation. The population within a radius of $1.61 \mathrm{~km}$ from NPPs received the highest TEDE of $760 \mathrm{mSv}$ and thus had the greatest excess cancer risk incidence rate. The infants had the greatest LAR of cancer incidence for all
TABle 1: Estimated TEDE with distance [14].

\begin{tabular}{lcccccccc}
\hline Distance $(\mathrm{km})$ & 1.61 & 2.41 & 3.22 & 4.83 & 6.44 & 16.1 & 24.1 & 32.2 \\
\hline TEDE $(\mathrm{mSv})$ & 760 & 340 & 190 & 87 & 51 & 23 & 17 & 16 \\
\hline
\end{tabular}

solid cancers, thyroid, and leukemia, respectively. The assessment of the cancer risk incidence in men was lower than females with solid cancers having the highest LAR $\left(10^{-2}\right)$ of 40.1. Leukemia LAR was greatest for males when compared with females. The risk for thyroid cancer incidence was greatest in female infants when compared with the male infants. Leukemia showed the lowest risk incidence in both sexes and age groups. The LAR of all solid cancers combined was estimated as the sum of all risks of individual organs. Generally, the cancer risk estimates were much higher for females than those for males, which might be due to the combined effects of the higher radio-sensitivity in women than in men.

3.3. Comparison of Lifetime Attributable Risk. Doses within a $20 \mathrm{~km}$ radius around Fukushima Daiichi NPPs had not been assessed in the WHO preliminary dose estimation, and therefore this geographical area was not included in this health risk assessment [7]. WHO only considered groups 1 and 2 for the risk assessment as shown in Table 4 . For the purpose of comparison, dose values estimated in this study that had a dose range of $12 \sim 25 \mathrm{mSv}$, which was similar to the dose range considered by WHO risk assessment in Group 1, were considered.

With the consideration of the mean, the estimated LAR at Fukushima Daiichi NPPs showed a variation by sex, age, and dose as shown in Figures 1 and 2. These doses were estimated by RASCAL for the first four days after the accident, whereas WHO considered a dose received in the first year to determine the cancer risk. According to WHO risk assessment, the LAR $\left(10^{-2}\right)$ for solid cancers in males and females ranged from $0.225 \sim 0.730$ and $0.336 \sim 1.113$, respectively. This study estimated the LAR $\left(10^{-2}\right)$ in the range of $0.223 \sim 0.668$ and $0.345 \sim 1.24$ for males and females, respectively. WHO estimated leukemia cancer incidence risk to be between $0.015 \sim 0.023$ and $0.009 \sim 0.016$ for males and females, respectively, for the case of leukemia, whereas the LAR $\left(10^{-2}\right)$ for leukemia in this study was estimated to be 0.0155 0.055 and 0.0118 0.0375 for males and females as shown in Figures 1 and 2, respectively. The LAR $\left(10^{-2}\right)$ for thyroid cancer risk in males and females ranged from $0.010 \sim 0.118$ and $0.048 \sim 0.524$, respectively, whereas an estimation for $\operatorname{LAR}\left(10^{-2}\right)$ in this study ranged from $0.0722 \sim 0.545$ and $0.0369 \sim 0.265$, respectively. The obtained results were compared with each other, and the comparison showed good agreement between this study and WHO report within the same order of magnitude $\left(10^{-2}\right)$ of cancer risk estimates although the observed differences might be attributed to many uncertainties of estimated doses and assumed population data. WHO applied the Japanese population status in 2004, whereas the population status of 2010 was used for this study. In addition, a sample size of 100,000 persons at exposure was applied in this study, while 
TABLE 2: LAR $\left(10^{-2}\right)$ of females per 100,000 exposed persons.

\begin{tabular}{|c|c|c|c|c|c|c|c|c|c|}
\hline \multicolumn{4}{|l|}{ Infants } & \multicolumn{3}{|c|}{ Children } & \multicolumn{3}{|c|}{ Adults } \\
\hline Distance $(\mathrm{km})$ & Solid cancers & Leukemia & Thyroid cancer & Solid cancers & Leukemia & Thyroid cancer & Solid cancers & Leukemia & Thyroid cancer \\
\hline 1.61 & 40.1 & 1.24 & 8.77 & 24.9 & 0.753 & 4.15 & 16.4 & 0.56 & 1.75 \\
\hline 2.41 & 18.3 & 0.554 & 3.92 & 11.2 & 0.337 & 1.86 & 7.33 & 0.25 & 0.785 \\
\hline 3.22 & 10.2 & 0.31 & 2.19 & 6.23 & 0.188 & 1.04 & 4.1 & 0.14 & 0.439 \\
\hline 4.83 & 4.67 & 0.142 & 1 & 2.85 & 0.0862 & 0.475 & 1.88 & 0.0641 & 0.201 \\
\hline 6.44 & 2.74 & 0.0831 & 0.588 & 1.67 & 0.0506 & 0.278 & 1.1 & 0.0376 & 0.118 \\
\hline 16.1 & 1.24 & 0.0375 & 0.265 & 0.755 & 0.0228 & 0.126 & 0.496 & 0.0169 & 0.0531 \\
\hline 24.1 & 0.913 & 0.0277 & 0.196 & 0.558 & 0.0169 & 0.0928 & 0.367 & 0.0125 & 0.0393 \\
\hline 32.2 & 0.86 & 0.0261 & 0.185 & 0.525 & 0.0159 & 0.0874 & 0.345 & 0.0118 & 0.0369 \\
\hline $\operatorname{LBR}\left(\times 10^{-2}\right)$ & 29.04 & 0.43 & 0.76 & 29.09 & 0.43 & 0.77 & 29.07 & 0.40 & 0.77 \\
\hline
\end{tabular}

TABLE 3: LAR $\left(10^{-2}\right)$ of males per 100,000 exposed persons.

\begin{tabular}{|c|c|c|c|c|c|c|c|c|c|}
\hline \multicolumn{4}{|l|}{ Infants } & \multicolumn{3}{|c|}{ Children } & \multicolumn{3}{|c|}{ Adults } \\
\hline Distance $(\mathrm{km})$ & Solid cancers & Leukemia & Thyroid cancer & Solid cancers & Leukemia & Thyroid cancer & Solid cancers & Leukemia & Thyroid cancer \\
\hline 1.61 & 22.1 & 1.82 & 1.8 & 15.2 & 0.963 & 0.853 & 10.6 & 0.739 & 0.366 \\
\hline 2.41 & 9.88 & 0.813 & 0.805 & 6.81 & 0.431 & 0.382 & 4.73 & 0.33 & 0.164 \\
\hline 3.22 & 5.52 & 0.454 & 0.45 & 3.81 & 0.241 & 0.213 & 2.64 & 0.185 & 0.0914 \\
\hline 4.83 & 2.53 & 0.208 & 0.206 & 1.74 & 0.11 & 0.0977 & 1.21 & 0.0845 & 0.0419 \\
\hline 6.44 & 1.48 & 0.122 & 0.121 & 1.02 & 0.0646 & 0.0573 & 0.709 & 0.0496 & 0.0245 \\
\hline 16.1 & 0.668 & 0.055 & 0.0545 & 0.461 & 0.0292 & 0.0258 & 0.32 & 0.0224 & 0.111 \\
\hline 24.1 & 0.494 & 0.0407 & 0.0403 & 0.341 & 0.0215 & 0.0191 & 0.236 & 0.0165 & 0.0818 \\
\hline 32.2 & 0.461 & 0.0383 & 0.0379 & 0.321 & 0.0203 & 0.00387 & 0.223 & 0.0155 & 0.0722 \\
\hline LBR $\left(\times 10^{-2}\right)$ & 40.74 & 0.57 & 0.21 & 40.71 & 0.58 & 0.21 & 40.60 & 0.60 & 0.21 \\
\hline
\end{tabular}

TABLE 4: Group classification considered by WHO study.

\begin{tabular}{lc}
\hline Group & Description \\
\hline Group 1 & Two locations in Fukushima prefecture with effective doses of $12 \sim 25 \mathrm{mSv}$ \\
Group 2 & Locations in Fukushima prefecture with effective doses of $3 \sim 5 \mathrm{mSv}$ \\
Group 3 & Less-affected locations in Fukushima prefecture and the rest of Japan with effective doses of 1 mSv \\
Group 4 & Neighboring countries and the rest of world with effective doses of well below of $1 \mathrm{mSv}$ \\
\hline
\end{tabular}

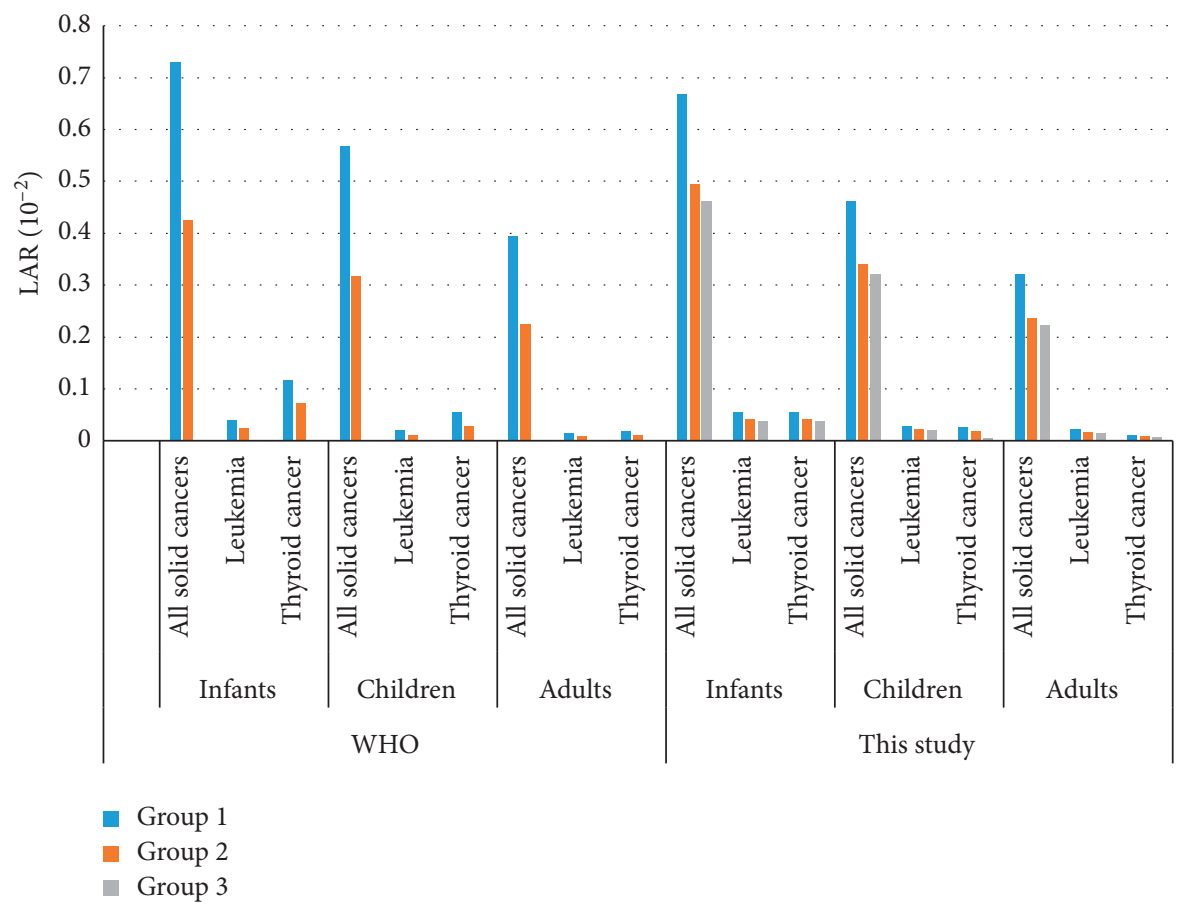

FIgURE 1: Comparison of LAR for cancer sites of males between WHO and this study. 


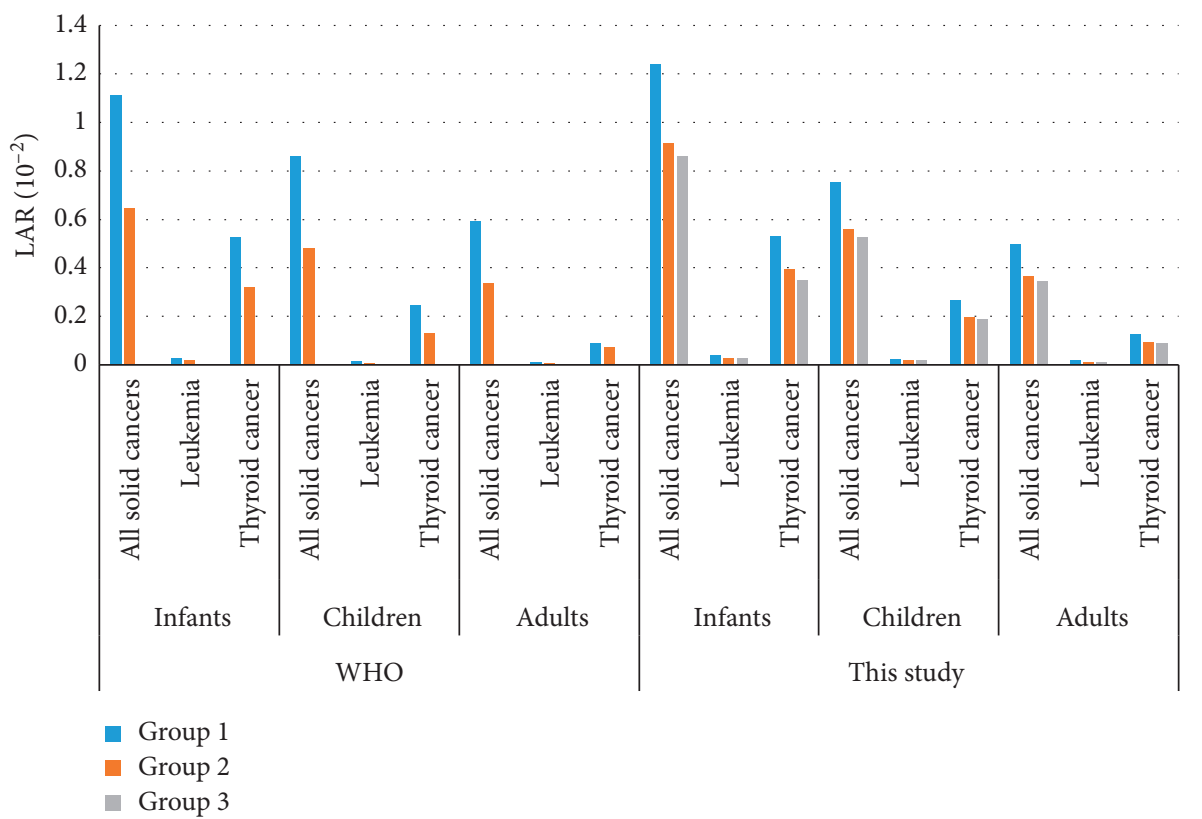

FIgURE 2: Comparison of LAR for cancer sites of females between WHO and this study.

10,000 persons were used for WHO risk assessment. Therefore, the proposed approach of coupling RASCAL and RadRAT in this study has proved applicable to the implementation of risk-informed decision-making supports for the responsible nuclear emergency organizations.

\section{Conclusions}

This study aimed at estimating the radiological risk associated with exposure to radioactive materials from Fukushima Daiichi NPPs accident. The estimates were compared with WHO estimates to ascertain their correlation and validity and reemphasize the need for understanding cancer risk assessment. It was assumed in this study that 100,000 persons of ages of 1 year, 10 years, and 20 years received a whole body dose at different distances from NPPs. LAR and the probability of an incidence of cancer attributable to radiation exposure from the Fukushima Daiichi NPPs accident in a representative member of the population in Japan were estimated in terms of excess cancer cases per 100,000 persons by sex, age, and distance at exposure. Using RASCAL, the source term and dose were determined by authors in the previous literature, and they were applied to assess the cancer risk incidences in this study. The source term was estimated to be $1.3 \times 10^{18} \mathrm{~Bq}$, whereas the TEDE was determined to be $16 \sim 760 \mathrm{mSv}$ in the radius of $1.61 \sim 32 \mathrm{~km}$ from NPPs. It was assumed that people in the most affected areas were within the radius of $32.2 \mathrm{~km}$; however, for the purpose of comparison with WHO risk estimates, only the population within $16.1 \sim 32.2 \mathrm{~km}$ were considered and estimated to receive a dose between 23 and $16 \mathrm{mSv}$. Lifetime cancer risks were determined using RadRAT online tool. For solid cancers, LAR $\left(10^{-2}\right)$ was estimated in a range of $0.223 \sim 0.668$ and $0.345 \sim 1.24$ for males and females, respectively, whereas the LAR $\left(10^{-2}\right)$ for leukemia was estimated at $0.0155 \sim 0.055$ and $0.0118 \sim 0.0375$ for males and females, respectively. LAR $\left(10^{-2}\right)$ for thyroid cancer ranged from $0.0722 \sim 0.545$ and $0.0369 \sim 0.265$, respectively. Solid cancer incidence risks were observed to be higher at younger ages-of-exposure and were somewhat higher in females than in males. For all the cancer risks, the most illustrative was thyroid cancer, with a significant evidence of higher risks at younger ages and with weak evidence for an excess or significantly lower risks following radiation exposure in adulthood. This study observed that age at the time of irradiation was one of the most important biological variables influencing cancer risk incidences. The results showed the highest excess cancer risks among those exposed in infancy (leukemia in males and solid cancers in females). The estimates in this study showed a significant correlation with WHO cancer risk estimates. It was found that the observed differences might be attributed to the variations in the methods, dose estimates, distances considered, and population status.

\section{Abbreviations}

BEIR: Biologic effects of ionizing radiation

CEDE: Committed effective dose equivalent

DDREF: Dose and dose-rate effectiveness factor

LAR: Lifetime attributable risk

LBR: $\quad$ Lifetime baseline risk

LSS: $\quad$ Life span study

NPP: Nuclear power plant

NRC: Nuclear regulatory commission

RadRAT: Radiation risk assessment tool

RASCAL: Radiological assessment system for consequence analysis

TEDE: Total effective dose equivalent

WHO: World Health Organization. 


\section{Data Availability}

The data used to support the findings of this study are included within the article.

\section{Conflicts of Interest}

The authors declare that they have no conflicts of interest.

\section{Acknowledgments}

This research was supported by the 2020 Research Fund of the KEPCO International Nuclear Graduate School (KINGS), the Republic of Korea. This research was also supported by the National Research Foundation of Korea (NRF) grant funded by the Korea government, Ministry of Science and ICT (No. NRF-2020M2A8A4022526).

\section{References}

[1] IAEA, The International Nuclear and Radiological Event Scale User's Manual, International Atomic Energy Agency, Vienna, Austria, 2013.

[2] C. Sofiya, "Deterministic and stochastic effects of radiation," Cancer Therapy and Oncology International Journal, vol. 12, no. 2, Article ID 555834, 2018.

[3] D. L. Preston, E. Ron, S. Tokuoka et al., "Solid cancer incidence in atomic bomb survivors: 1958-1998," Radiation Research, vol. 168, no. 1, pp. 1-64, 2007.

[4] ICRU, "Major releases of radionuclides to the environment," Journal of International Commission on Radiation Units and Measurements, vol. 15, no. 1-2, pp. 4-36, 2019.

[5] G. Smith, "UNSCEAR 2013 Report. Volume I: report to the general assembly, Annex A: levels and effects of radiation exposure due to the nuclear accident after the 2011 great eastJapan earthquake and tsunami," Journal of Radiological Protection, vol. 34, no. 3, pp. 725-727, 2014.

[6] L. Walsh, W. Zhang, R. E. Shore et al., "A framework for estimating radiation-related cancer risks in Japan from the 2011 Fukushima nuclear accident," Radiation Research, vol. 182, no. 5, pp. 556-572, 2014.

[7] WHO, Risk Assessment from the Nuclear Accident after the 2011 Great East Japan Earthquake and Tsunami Based on a Preliminary Dose Estimation, World Health Organization, Geneva, Switzerland, 2013.

[8] The National Academy of Sciences, Lessons Learned from the Fukushima Nuclear Accident for Improving Safety of U.S. Nuclear Plants, The National Academy Press, Washington, DC, USA, 2014.

[9] EPRI, Fukushima Radiological Assessment Tool, Version 2: Benchmarking Radiological Assessment and Dose Models Using Fukushima Dataset, Electric Power Research Institute, Palo Alto, CA, USA, 2014.

[10] J. V. Ramsdell, G. F. Athey, S. A. McGuire, and L. K. Brandon, RASCAL 4: Description of Models and Methods, NUREG-1940, U.S. Nuclear Regulatory Commission, Washington, DC, USA, 2012.

[11] J. V. Ramsdell, G. F. Athey, and J. P. Rishel, RASCAL 4.3: Description of Models and Methods, NUREG-1940 Supplement 1, U.S. Nuclear Regulatory Commission, Washington, DC, USA, 2015.

[12] National Cancer Institute, https://radiationcalculators.cancer. gov/radrat/about/, 2020.
[13] A. B. de Gonzalez, A. Iulian Apostoaei, L. H. S. Veiga et al., "A radiation risk assessment tool for lifetime cancer risk projection," Journal of Radiological Protection, vol. 32, no. 3, pp. 205-222, 2012.

[14] F. Rukundo and J. Kim, "Radiological environmental consequence analysis of hypothetical severe accidents at nuclear power plants," Journal of Korean Society of Environmental Engineers, vol. 41, no. 8, pp. 440-450, 2019.

[15] The National Academy of Sciences, Health Risks from Exposure to Low Levels of Ionizing Radiation: BEIR VII Phase 2, The National Academy Press, Washington, DC, USA, 2006. 